so far in the other direction, that psychiatrists find it hard to prescribe the medication even to those who will benefit from its use. ${ }^{5}$ Second, although the main push seems to be towards reduction of antipsychotic use, less is said on how to develop the resources that can provide good non-pharmacological approaches. Audits and targets should not solely focus on the quantity of antipsychotic use in dementia but also on the quality of non-pharmacological approaches available to this population. Long strides are required in this direction to improve behavioural and psychological symptoms of dementia care in the community, hospitals and care homes.

1 Treloar A, Crugel M, Prasanna A, Solomons L, Fox C, Paton C, et al. Ethical dilemmas: should antipsychotics ever be prescribed for people with dementia? Br J Psychiatry 2010; 197: 88-90.

2 Hope J. More than 100,000 dementia patients are given anti-psychotic drugs that 'may kill'. Mail Online 2008; 28 April.

3 Laurance J. 'Liquid cosh' treatment kills dementia patients. The Independent 2009; 9 January

4 Triggle N. Dementia drug use 'killing many'. BBC News 2009; 12 November.

5 Brummel-Smith $\mathrm{K}$. It's time to require written informed consent when using antipsychotics in dementia. Br J Med Pract 2008; 1: 4-6.

Rajesh Sekhri, Garnock Day Centre, Ayrshire Central Hospital, Irvine KA12 8SS, UK. Email: rajeshsekhri@nhs.net

doi: 10.1192/bjp.197.6.501a

Authors' reply: We are pleased to see support for our views in the responses received, in particular for the focus on rational debate in this area, rather than an 'either/or' debate. However, we would in reply air the following cautions.

Pattanayak claims that antipsychotics may not be as harmful as we have been led to believe. We would urge caution here. The data produced so far do suggest a reliable and quantifiable degree of harm resulting from antipsychotic use. We do not think that it is truly reasonable, now, to suggest that these drugs may be harmless. But we would also argue that Pattanayak's desire to claim a lack of harm is unnecessary. If they are harmful, their use may be justified under the doctrine of double effect by balancing the likely benefits against harm. It is our clinical experience that the discussions of benefit versus harm with relatives and advocates, informed by the principal of double effect, are effective and well understood.

Finally, we note what is, perhaps, an important slip of the pen in Sekhri's letter. He uses the term non-pharmacological as shorthand for alternatives to antipsychotics. He is correct in saying that there are multiple causes of behavioural and psychological symptoms of dementia. But if we describe all alternatives to antipsychotics as non-pharmacological, we may forget the appropriate treatment of physical illness with analgesics or depression with antidepressants. Alternatives to antipsychotic use in distress include both pharmacological and non-pharmacological approaches. One of us remembers replacing haloperidol for behavioural and psychological symptoms of dementia with effective treatment for scabies. Aromatherapy would have done little here! The term 'non-pharmacological' may therefore distract clinicians and compound a paucity of response to a complex problem.

Adrian J. Treloar, Oxleas NHS Foundation Trust, Memorial Hospital, Shooters Hill, London SE18 3RG, UK. Email: adrian.treloar@oxleas.nhs.uk; Carol Paton, Oxleas NHS Foundation Trust, Imperial College, London, and the Prescribing Observatory for Mental Health; Monica Crugel, Oxleas NHS Foundation Trust, London; Luke Solomons, South London and Maudsley NHS Foundation Trust; Aparna Prasanna, Hospital of St Cross, Coventry and Warwickshire Partnership NHS Trust, Rugby; Chris Fox, Kent and Medway NHS \& Social Care Partnership Trust, UK

doi: 10.1192/bjp.197.6.502

\section{Interventions for self-harm: are we measuring outcomes in the most appropriate way?}

Kapur and colleagues provide a brief review of contact-based interventions for self-harm and note their continuing appeal, despite largely unconvincing trial results. ${ }^{1}$ The question the authors should have posed is not 'How might these interventions work?' but 'Why, when participants report that the interventions are of benefit, are trial results so unconvincing?'

The importance of self-harm lies in its strong association with suicide. The ultimate aim of interventions in this area is to prevent suicide, but the rarity of suicide makes it difficult to use as an outcome measure. Of those studies reviewed by Kapur et al, only two used death as an outcome. ${ }^{2,3}$ The remainder used repetition of self-harm, which is the best available proxy measure. ${ }^{4}$

Measuring repetition of self-harm is problematic. Hospitaltreated episodes represent the standard measure but fail to capture the true pattern of self-harming behaviour, most of which occurs in secret and does not result in hospital presentation. Those who repeatedly self-harm avoid accident and emergency (A\&E) departments at all costs and, when forced by the severity of their injuries to present, are adept at concealing the self-inflicted nature of those injuries, resulting in possible miscoding of visits. There is a need for a reliable, user-designed self-report instrument and a better understanding of the relationship between acts of self-harm and hospital visits.

Hospital-treated episodes do not provide a measure of reduction in self-harm; only a measure of reduction in clinical encounters for self-harm. It is debatable whether reducing clinical encounters is a beneficial outcome for this highly vulnerable and hard-to-reach population (repeat self-harmers). Reducing the number of hospital presentations may cut service costs in the short term; it may not save lives.

In a recent pilot study of a text-messaging intervention for self-harm, ${ }^{5}$ we had an interesting case. One of the participants reported during the trial that the intervention (a text message) had saved their life by interrupting a suicide attempt and prompting them to call for help instead of taking an overdose. They were conveyed to $\mathrm{A} \& \mathrm{E}$ and treated for very severe lacerations. Partly as a result, their visits to A\&E increased during the 6-month pilot trial compared with 6 months prior to entry: a negative result using hospital-treated episodes as a measure. Two further participants told us that a suicide attempt had been interrupted by the timely arrival of a text message and begged to be allowed to continue to use the intervention at the end of the trial, yet standard reporting of the results of the study would not provide convincing evidence of effectiveness.

So why are trial results unconvincing, despite qualitative evidence to the contrary? The low status of qualitative data is one possible reason. Another is that we are measuring outcomes in inappropriate ways. We do not yet understand what outcomes are important to those who engage in repeated self-harm, nor how best to measure them.

1 Kapur N, Cooper J, Bennewith O, Gunnell D, Hawton K. Postcards, green cards and telephone calls: therapeutic contact with individuals following selfharm. Br J Psychiatry 2010; 197: 5-7.

2 Motto JA, Bostrom AG. A randomized controlled trial of postcrisis suicide prevention. Psychiatr Serv 2001; 52: 828-33.

3 Fleischmann A, Bertolote J, Wasserman D, De Leo D, Bolhari J, Botega NJ, et al. Effectiveness of brief intervention and contact for suicide attempters: a randomized controlled trial in five countries. Bull World Health Organ 2008; 86: 703-9.

4 Hawton K, Arensman E, Townsend E, Bremner S, Feldman E, Goldney R, et al. Deliberate self-harm: systematic review of efficacy of psychosocial and pharmacological treatments in preventing repetition. BMJ 1998; 317: 441-7. 
5 Owens C, Farrand P, Darvill R, Emmens T, Hewis E, Aitken P. Involving service users in intervention design: a participatory approach to developing a text-messaging intervention to reduce repetition of self-harm. Health Expect 2010; doi: 10.1111/j.1369-7625.2010.00623.x. Epub ahead of print.

Christabel Owens, Devon Partnership NHS Trust, and Peninsula Medical Schoo University of Exeter, Veysey Building, Salmon Pool Lane, Exeter EX2 4SG, UK. Email: christabel.owens@pms.ac.uk

doi: 10.1192/bjp.197.6.502a

Authors' reply: We agree with much of what Dr Owens says, but take issue with three points.

First, we disagree that we asked the wrong question. The possibility that a brief message of concern from toxicologists that the individuals may never have met might halve the number of repeat episodes of self-harm is intriguing. ${ }^{1}$ Of course we need to ask what the possible mechanisms might be.

Second, self-harm greatly increases the risk of suicide but it is much more than a proxy measure in trials. As clinicians working in accident and emergency departments and mental health settings will testify, self-harm is important in its own right - there may over 200000 hospital presentations for self-harm in England every year. $^{2}$

Third, although we are all fans of qualitative research and the additional insights it provides, the main reason for negative trial results is not the low status of qualitative data. Negative findings are more likely to reflect the fact that trials to date have been too small to detect clinically important effects ${ }^{3}$ (or alternatively that the interventions simply do not work).

Outcomes for trials are definitely an issue and Dr Owens summarises a number of the key considerations. Many studies to date have used repeat episodes of self-harm presenting to hospital as the principal outcome measure. We did argue (perhaps somewhat clumsily) in an earlier version of our article that such repeat presentations might actually be an indication of positive engagement with services. We deleted the offending passage following editorial and reviewers' comments. The case study that Owens briefly presents is very interesting and of course would not be picked up by standard reporting of trial results. Using qualitative data to comprehensively measure outcomes on all participants in large trials is impractical. A challenge for selfreport measures may be the painfully low response rates. However, we would support Dr Owens' call for a variety of outcome measures - hospital-based and self-report, quantitative and qualitative.

\section{Declaration of interest}

N.K. is Chair of the Guideline Development Group for the forthcoming National Institute for Health and Clinical Excellence (NICE) guideline on the longer-term management of self-harm. The views expressed in this letter are those of the authors and not those of the Guideline Development Group, NICE or the National Collaborating Centre for Mental Health.

1 Carter GL, Clover K, Whyte IM, Dawson AH, D'Este C. Postcards from the EDge: 24-month outcomes of a randomised controlled trial for hospitaltreated self-poisoning. Br J Psychiatry 2007; 191: 548-53.

2 Hawton K, Bergen H, Casey D, Simkin S, Palmer B, Cooper J, et al. Self-harm in England: a tale of three cities. Multicentre study of self-harm. Soc Psychiatry Psychiatr Epidemiol 2007; 42: 513-21.

3 National Collaborating Centre for Mental Health. Self-harm: The Short-term Physical and Psychological Management and Secondary Prevention of Selfharm in Primary and Secondary Care. Clinical Guideline 16. The British Psychological Society \& The Royal College of Psychiatrists, 2004.

Navneet Kapur, Centre for Suicide Prevention, School of Community Based Medicine, University Place, University of Manchester, Oxford Road, Manchester M13 9PL, UK. Email: nav.kapur@manhester.ac.uk; Jayne Cooper, Centre for suicide Prevention, University of Manchester; Olive Bennewith, David Gunnell, Department of Social Medicine, University of Bristol; Keith Hawton, Centre for Suicide Research, University of Oxford, UK

doi: 10.1192/bjp.197.6.503

\section{Correction}

Failure to communicate effectively or failure of feedback? (letter). BJP, 197, 332-333. The first author's name is Raman D. Pattanayak. The online version of this letter has been corrected in deviation from print and in accordance with this correction.

doi: 10.1192/bjp.197.6.503a 